Annals of International Medical and Dental Research

E-ISSN: 2395-2822 | P-ISSN: 2395-2814

Vol-8, Issue-1 | January-February 2022

DOI: 10.53339/aimdr.2022.8.1.5

Page no- 31-37 | Section- Research Article (Microbiology)

\title{
Evaluation of Pathogens Causing Urinary Tract Infection and Their Sensitivity Pattern
}

\author{
Vijay Kumar1*, Swayambhu Shubham², Satyendra Narayan Singh ${ }^{3}$
}

\begin{abstract}
${ }^{1}$ Associate Professor, Department of Microbiology, Patna Medical College and Hospital, Patna, Bihar, India.

Email: drkvijay10@yahoo.com Orcid ID: 0000-0002-6978-8102

23rd year P.G, Department of Radiodiagnosis, Narayan Medical College and Hospital, Jamuhar, Sasaram, Bihar, India.

Email: drshubham97@gmail.com

Orcid ID: 0000-0002-3969-536X

3Professor, Department of Microbiology, Patna Medical College and Hospital, Patna, Bihar, India.

Email: drsnsinghpmch@gmail.com

Orcid ID: 0000-0003-1004-7868
\end{abstract}

*Corresponding author

Received: 04 August 2021

Revised: 30 September 2021

Accepted: 09 October 2021

Published: 22 December 2021

\begin{abstract}
Background: UTI constitute a major public health problem in India accounting 2nd most common infection next to respiratory tract infection. They are responsible for increasing treatment cost and significant morbidity. Aim:- To determine the incidence of UTI, evaluation of pathogens responsible and their antimicrobial susceptibility pattern in the population. Methods: Urine samples were collected from 300 patients attending the OPD Patna medical college, Patna during the period of 18 months (January 2017 to June 2018) Antimicrobial sensitivity testing was done for the bacterial isolates present in the sample by Kirby- Bauer disc diffusion method. Only those samples were taken into consideration which develops count equal to or greater than $1 * 10^{5} \mathrm{CFU} / \mathrm{ml}$ as indicated by Kass. Results: Out of 300 samples collected 146 (48.66\%)) yielded bacterial growth. Out of 146 culture isolates E.Coli was the most common pathogen followed by klebsiella, CoNS and staphylococcus. Antibiotic sensitivity was performed on all the isolates. It was observed that highest sensitivity was $49.31 \%$ to amikacin, gentamycin (45.89\%), nitrofurantoin $(38.35 \%)$ meropenem $(27.39 \%)$. Conclusions: It was observed that high grade of resistance to ampicillin, cotrimoxazole, ciprofloxacin, cefuroxime, chloramphenicol, cefotaxime, cefazolin, amoxicillin + clavulanic acid and gentamycin is present as a result of misuse or improper use of antibiotic in the community. Hence urine culture is necessary for the diagnostic screening of UTI before the treatment.
\end{abstract}

Keywords:- Urinary tract Infection (UTI), Coagulase Negative Staphylococcus (CoNS), Outdoor Patients Department (OPD), Antimicrobial Sensitivity Susceptibility Tests (AST).

\section{INTRODUCTION}

UTI is defined as a disease caused by microbial invasion of the urinary tract that extends from the renal cortex of the kidneys to the urethral meatus. This accounts for the $2^{\text {nd }}$ most common infection after respiratory tract infection in the community and encountered by medical practitioners in routine practice. ${ }^{11}$ UTI is the term used to describe acute urethritis and cystitis caused by microorganisms. It accounts for $1-3 \%$ of consultation in general practice.

The prevalence of UTI in women is about $3 \%$ at the age of 20 , increasing by about $1 \%$ in each subsequent decade goes upto $20 \%$. More than half of all healthy women experience at least 1 symptomatic UTI in their lifetime and each year $2-10 \%$ of the women experience one episode. ${ }^{[2,3,4]}$ In women the ascent of organism 
Annals of International Medical and Dental Research E-ISSN: 2395-2822 | P-ISSN: 2395-2814 Vol-8, Issue-1 | January-February 2022 DOI: $10.53339 /$ aimdr.2022.8.1.5

Page no- 31-37 | Section- Research Article (Microbiology)

into the bladder is easier than men due to the short urethra and absence of bactericidal prostatic secretions. It is not so common in males with normal genito-urinary tract but increases after the age of 65 yrs due to prostatic hypertrophy and prostatitis. Every year more than 150 million people gets infected worldwide and causes a great economical burden. $[\underline{[5,6,7]}$

Clinical presentation in general are abrupt onset of frequency of micturition, urgency, dysuria, suprapubic pain during and after micturition, intense desire to pass more urine after micturition due to inflamed bladder, appearance of cloudy urine with odour. [8,9] Upper UTI involves kidney and ureter causing pyelonephritis and ureteritis, perinephric abscesses, renal abscess whereas lower UTI causes asymptomatic bacteriuria, cystitis, urethritis and acute urethral syndrome.Gender, sexual activity, pregnancy, obstruction to flow due to any reason, neurogenic bladder dysfunction, vesico-ureteric reflux and genetic factor plays an important role in UTI.

E.Coli is the most common isolate in both the outdoor and indoor patients accounting for 75$90 \%$ of uncomplicated UTI. The less commonly isolated organisms are $S$. saprophyticus, Klebsiella, Proteus, Enterococcus and Enterobacter. UTI has been associated with significant morbidity, development of renal scarring and later to renal failure, hypertension and eclampsia. 10] Scheffer in 2002 and Bishwas in 2006 advocated the preference for Fluoroquinolone as initial drug for empirical treatment of UTI where resistance is a matter of concern because of their high bacteriological and clinical cure rate and low rate of resistance to common organisms. [11,12] In a study done by kulkarny on 1000 samples 395 were positive for E.coli and on antibiotic susceptibility test majority of isolates were turned resistant to most of the commonly used antibiotics for UTI treatment.

In recent years the development of antibiotics resistant has become a major problem worldwide. It's the result of extensive use of antimicrobial. The microorganism and their resistance to the antimicrobial has been changing continuously in the society.

In India there is a common practice of medical practitioners to prescribe broad spectrum antibiotics for empirical treatment before getting culture and sensitivity report of the urine sample. So area wise surveillance of microorganism causing UTI and their antimicrobial sensitivity is essential for selecting and appropriate antibiotic for treatment and prevent the antimicrobial resistance. The resistance pattern of UTI pathogens have not been extensively studied.[12] So there is need for study on pathogens and their sensitivity pattern isolated from UTI cases in India.

This retrospective study was done to evaluate the microorganism responsible for causing UTI and their sensitivity/resistance pattern in the patients of UTI in Bihar, India.

\section{MATERIAL AND METHODS}

Selection of participants - Urine samples were collected from 300 patients with symptoms of UTI attending the OPD of Patna medical college, Patna during the period of 18 months (Jan 2017 to June 2018) . 
Annals of International Medical and Dental Research

E-ISSN: 2395-2822 | P-ISSN: 2395-2814

Vol-8, Issue-1 | January-February 2022

DOI: $10.53339 / a i m d r .2022 .8 .1 .5$

Page no- 31-37 | Section- Research Article (Microbiology)

Preparation of materials- All the media in the experiment as nutrient agar (NA), Mac-Conkey agar, blood agar and Muller Hinton agar were prepared in sterilized manner supplied by $\mathrm{Hi}$ media laboratory. The glassware including petri dishes were sterilized in regular manner by autoclaving during the period.

Collection of urine samples- participants were directed to take clean voided midstream urine specimen after cleaning the meatus in the sterilized container made ready to use for the patients in the microbiology lab. There were no matter of transport because all facilities were available in the lab itself.

Isolation of bacteria- under the biosafety cabinet after dilution of the specimen in normal saline the specimen was added in the molten agar after being cooled and solidified. The petri dish were incubated at $37^{\circ} \mathrm{C}$. After $24 \mathrm{hrs}$ of incubation bacterial colonies appear on the plate and counted for detection of significant bacteuria. Then a loop full of each sample were smeared on to solid media (Mac Conkey and blood agar plate) by streaking method of culture for isolation of bacteria in the urine sample and inoculated culture plates were incubated at $37^{\circ} \mathrm{c}$ aerobically overnight in the incubator. On the next day selection of plates with growth were done and subculture of the growth on agar were done for the purpose of identification of bacteria.

Identification of bacteria- after incubation culture media were taken out of incubator and examined in open light to see the appearance of bacterial colonies (size, shape, consistency, density, color of colony on different culture media and pigment production if any in the media and of any odor). This was followed by
Grams staining and motility test under the microscope and lastly a battery of biochemical test as catalase test, oxidase test, indole test, citrate utilization, urease test, triple sugar iron agar test $\mathrm{H}_{2} \mathrm{~S}$ production were applied.

Antimicrobial susceptibility test (AST)- Kirby - Bauer's disc diffusion test (1966) was used for antimicrobial sensitivity. The turbidity of the inoculum were adjusted to $0.5 \mathrm{Mac}$ Farland opacity standard which is equivalent to $1.5^{\star} 10^{8}$ $\mathrm{CFU} / \mathrm{ml}$ of bacteria by inoculating the test organism in broth solution followed by inoculating at $37^{\circ} \mathrm{C}$ for $2-4 \mathrm{hrs}, 0.1 \mathrm{ml}$ of broth is inoculated on the surface of culture media by steaking with sterile cotton swab, left for 10 minutes. Then antibiotic impregnated $6 \mathrm{~mm}$ diameter filter paper disc (Hi media) were dispensed with dispenser on to the media streaked with isolates and the reading were taken after incubating the plate for $24 \mathrm{hrs}$ at 370 $C$ aerobically. Next day the diameter of zone of inhibition was measured and compared with the zone diameter interpretation chart provided by $\mathrm{Hi}$ media and the result as sensitivity for resistance of the isolated bacteria to antibiotics are determined.

\section{RESULTS}

Total 300 samples were included in this study. Data were collected and presented on the tables. [Table 1] shows the total number of samples included in the test $(n=300)$. Out of which male samples were 81 and among which 27 developed growth. Among the total 219 female samples 119 developed the growth of bacteria, mixed growth developed in 16 samples. Total 138 samples (85 female and 53 male) did not develop any growth. 
Annals of International Medical and Dental Research

E-ISSN: 2395-2822 | P-ISSN: 2395-2814

Vol-8, Issue-1 | January-February 2022

DOI: 10.53339/aimdr.2022.8.1.5

Page no- 31-37 | Section- Research Article (Microbiology)

[Table 2] shows the microorganism responsible for causing UTI in the population. This table shows the most prevalent and highly responsible organism for UTI in the population was E.Coli in the study causing total $36.98 \%$ of infections so E.Coli is responsible for causing UTI in $36.98 \%$ of the population. Klebsiella is responsible for $3.42 \%$ of male and $17.12 \%$ of female population for causing UTI. This causes 2nd highest cases of UTI after E.Coli. CoNS is responsible for causing $13.69 \%$ (2.73\% in male and $10.95 \%$ in female) while staph. aureus is responsible for $8.90 \%$ of population. Other bacteria isolated from the samples responsible for UTI in the population are enterococcus $(5.47 \%)$, Acinetobacter $(5.47 \%)$, Citrobacter $(3.42 \%)$, proteus $(2.73 \%)$ and pseudomonas $(1.36 \%)$.
[Table 3] shows reports of antimicrobial sensitivity test in the samples. On observation of the sensitivity pattern shown by the organism it is seen that they were sensitive $49.31 \%$ to amikacin, $45.89 \%$ to gentamycin, $38.35 \%$ to nitrofurantoin, $27.39 \%$ to meropenem, $27.39 \%$ to piperacillin tazobactum, $26.71 \%$ to cefoperazone sulbactum, $20.45 \%$ to ciprofloxacin. It was observed that some drugs for which the population has shown their resistance as amikacin $10.95 \%$, gentamycin $20.54 \%$, nitrofurantoin $8.90 \%$, amoxicillin $24.65 \%$, ciprofloxacin $37.67 \%$ and others as depicted in the table. So it was seen that at the same time there are some common drugs to which the population is resistant and sensitive to other drugs.

Table 1: Sex Wise Distribution of Samples in Study Group

\begin{tabular}{|l|l|l|l|}
\hline & Male $(\mathbf{n = 8 1}) \mathbf{( 2 7 \% )}$ & Female $(\mathbf{n = 2 1 9}) \mathbf{( 7 3 \% )}$ & Total (n=300) (100\%) \\
\hline Sterile & $53(17.66 \%)$ & $85(28.34 \%)$ & $138(46 \%)$ \\
\hline Mixed & $01(0.33 \%)$ & $15(4.5 \%)$ & $16(4.83 \%)$ \\
\hline Positive & $27(9 \%)$ & $119(39.66 \%)$ & $146(48.66 \%)$ \\
\hline
\end{tabular}

Table 2: Organisms isolated in the urine samples of study group.

\begin{tabular}{|l|l|l|l|}
\hline & Male & Female & Total \\
\hline Organism & $27(9 \%)$ & $119(39.66 \%)$ & $146(48.66 \%)$ \\
\hline E.coli & $9(6.16 \%)$ & $45(30.82 \%)$ & $54(36.98 \%)$ \\
\hline Klebsiella & $5(3.42 \%)$ & $25(17.12 \%)$ & $30(20.54 \%)$ \\
\hline CoNS & $4(2.73 \%)$ & $16(10.95 \%)$ & $20(13.69 \%)$ \\
\hline Staphylococcus & $2(1.36 \%)$ & $11(7.53 \%)$ & $13(8.90 \%)$ \\
\hline Enterococcus & $2(1.36 \%)$ & $6(4.10 \%)$ & $8(5.47 \%)$ \\
\hline Acinetobacter & $2(1.36 \%)$ & $6(4.10 \%)$ & $8(5.47 \%)$ \\
\hline Citrobacter & $1(0.68 \%)$ & $4(2.73 \%)$ & $5(3.42 \%)$ \\
\hline Candida albicans & 0 & $2(1.36 \%)$ & $2(1.36 \%)$ \\
\hline Proteus & $1(0.68 \%)$ & $3(2.05 \%)$ & $4(2.73 \%)$ \\
\hline Pseudomonas & $1(0.68 \%)$ & $1(0.68 \%)$ & $2(1.36 \%)$ \\
\hline
\end{tabular}


Annals of International Medical and Dental Research E-ISSN: 2395-2822 | P-ISSN: 2395-2814

Vol-8, Issue-1 | January-February 2022

DOI: 10.53339/aimdr.2022.8.1.5

Page no- 31-37 | Section- Research Article (Microbiology)

Table 3: Antibiotic Sensitivity Pattern Of Organism Isolated From Study Groups

\begin{tabular}{|l|l|l|l|l|}
\hline \multirow{2}{*}{ Antibiotics } & \multicolumn{2}{|l|}{ Sensitivity pattern } & \multirow{2}{*}{ Total } \\
\cline { 2 - 4 } & $\mathbf{N R}$ & $\mathbf{R}$ & $\mathbf{S}$ & \\
\hline AMIKACIN & 58 & $16(10.95 \%)$ & $72(49.31 \%)$ & $146(100 \%)$ \\
\hline GENTAMYCIN & 49 & $30(20.54 \%)$ & $67(45.89 \%)$ & $146(100 \%)$ \\
\hline NITROFURANTOIN & 77 & $13(8.90 \%)$ & $56(38.35 \%)$ & $146(100 \%)$ \\
\hline AMOXYCILLIN & 74 & $36(24.65 \%)$ & $36(24.65 \%)$ & $146(100 \%)$ \\
\hline MEROPENAM & 96 & $10(6.8 \%)$ & $40(27.39 \%)$ & $146(100 \%)$ \\
\hline PIPERACILLIN+ TAZOBACTUM & 95 & $11(7.53 \%)$ & $40(27.39 \%)$ & $146(100 \%)$ \\
\hline CEFOPERAZONE+ SULBACTAM & 95 & $12(8.21 \%)$ & $39(26.71 \%)$ & $146(100 \%)$ \\
\hline COTRIMOXAZOLE & 56 & $54(36.98 \%)$ & $36(24.65)$ & $146(100 \%)$ \\
\hline CIPROFLOXACIN & 61 & $55(37.67 \%)$ & $30(20.45 \%)$ & $146(100 \%)$ \\
\hline LINEZOLID & 111 & $06(4.10 \%)$ & $29(19.86 \%)$ & $146(100 \%)$ \\
\hline CEFAZOLINE & 84 & $33(22.60 \%)$ & $29(19.86 \%)$ & $146(100 \%)$ \\
\hline CEFOTAXIME & 83 & $34(23.28 \%)$ & $29(19.86 \%)$ & $146(100 \%)$ \\
\hline CEFUROXINE & 83 & $36(24.65 \%)$ & $27(18.49 \%)$ & $146(100 \%)$ \\
\hline CHLORAMPHENICOL & 85 & $35(23.97 \%)$ & $26(17.80 \%)$ & $146(100 \%)$ \\
\hline TEICOPLANIN & 115 & $10(6.84 \%)$ & $21(14.38 \%)$ & $146(100 \%)$ \\
\hline CLINDAMYCIN & 106 & $21(14.38 \%)$ & $19(13.01 \%)$ & $146(100 \%)$ \\
\hline ERYTHROMYCIN & 103 & $25(17.12 \%)$ & $18(12.32 \%)$ & $146(100 \%)$ \\
\hline AMPICILLIN & 83 & $50(34.24 \%)$ & $13(8.90 \%)$ & $146(100 \%)$ \\
\hline NETLIMYCIN & 131 & $06(4.10 \%)$ & $09(6.16 \%)$ & $146(100 \%)$ \\
\hline NALIDIXIC ACID & 135 & $04(2.73 \%)$ & $07(4.79 \%)$ & $146(100 \%)$ \\
\hline TETRACYCLIN & 145 & $01(0.68 \%)$ & $00(0 \%)$ & $146(100 \%)$ \\
\hline
\end{tabular}

\section{DISCUSSION}

This study was done to determine the incidence of UTI in the population at Bihar, as well as to evaluate the microorganism involved in UTI. Out of 300 patients included in the study, pathogens were isolated in only 146 (27 males and 119 females). The study shows large number of organism with high bacterial count in female patients in comparison to males. In this study the incidence of growth is seen in 27 (9\%) males and 119 (39.66\%) females which is very much similar to the study showing high incidence of UTI in females with influence on direction of collection of samples to females.
The other cause may be the close proximity of urethra to the anus. Also a short incompetent urethra may be a cause in school girls, alterations in the vaginal flora promotes vaginal colonization and cause UTI.

In this study most commonly isolated pathogen was E.Coli which is similar to the other studies. In their study E.Coli was responsible for 52\% cases of UTI, klebsiella $14 \%$ and enterococcus $4 \%$ with high percentage in females. also found the E.Coli as the most prevalent bacterial species accounting for $60.3 \%$.

The result of the study shows high sensitivity of the isolated organism to amikacin (49.31\%), 
Annals of International Medical and Dental Research E-ISSN: 2395-2822 | P-ISSN: 2395-2814 Vol-8, Issue-1 | January-February 2022 DOI: $10.53339 /$ aimdr.2022.8.1.5

Page no- 31-37 | Section- Research Article (Microbiology)

gentamycin $(45.89 \%)$, nitrofurantoin (38.35\%) meropenem $(27.39 \%)$ cefoperazone sulbactum $(26.71 \%)$, piperacillin tazobactum $(27.39 \%)$, amoxicillin clavulanic acid (24.65\%), cotrimoxazole $(24.65 \%)$. It is seen that sensitivity to nitrofurantoin has got reduced, as opposed to the findings of WHO reported its higher efficacy against E.Coli, like that reported $57.9 \%$, sensitivity to nitrofurantoin. In this study amikacin was seen to be highest sensitive.

The international trend of treatment of UTI empirically may not apply to specific region like India where decreased sensitivity rates are documented for pathogens, so in India routine culture of urine may be necessary and specific guidelines should be provided, with the help of regional surveillance and getting information about current situation of sensitivity.

\section{CONCLUSIONS}

This study determines the incidence of urinary tract infection in the studied population. It also evaluates the major causative bacteria involved in UTI. The sensitivity pattern of the isolated

\section{REFERENCES}

1. Najar MS, Saldanha CL, Banday KA. Approach to urinary tract infections. Indian J Nephrol. 2009 Oct;19(4):129-39. doi: 10.4103/0971-4065.59333.

2. Nicolle L, Anderson PA, Conly J, Mainprize TC, Meuser J, Nickel JC, ET AL. Uncomplicated urinary tract infection in women. Current practice and the effect of antibiotic resistance on empiric treatment. Can Fam Physician. 2006;52(5):612-8.

3. Foxman B, Barlow R, D'Arcy H, Gillespie B, Sobel JD. Urinary tract infection: self-reported incidence and associated costs. Ann Epidemiol. 2000;10(8):509-15. doi: 10.1016/s1047-2797(00)00072-7.

4. Al-Badr A, Al-Shaikh G. Recurrent Urinary Tract Infections Management in Women: A review. Sultan organism in the UTI cases is comparable with other reported patterns with E.Coli being the most common causative organism in UTI. This is followed by klebsiella, CoNS. This study shows a high rate of sensitivity to amikacin $(49.31 \%)$, gentamycin $(45.89 \%)$, nitrofurantoin $(38.35 \%)$ meropenem (27.39\%). These drugs should be considered as 1st line drugs for treating the cases of UTI in Bihar. However the study shows a high grade of resistance to ampicillin, cotrimoxazole, ciprofloxacin, cefuroxime, chloramphenicol, cefotaxime, cefazolin, amoxicillin clavulanic acid and gentamycin. This denotes the misuse or inappropriate use of these commonly used antibiotics in empirical treatment. So the culture of urine sample is extremely recommended as the screening method before starting the treatment, as most of the cases of UTI are asymptomatic in early stage before development of severe complications. Routine screening of febrile patients should be done to administer proper antibiotic in time, by sensitivity testing to prevent the complications.

Qaboos Univ Med J. 2013;13(3):359-367. doi:10.12816/0003256

5. Brumbaugh AR, Mobley HL. Preventing urinary tract infection: progress toward an effective Escherichia coli vaccine. Expert Rev Vaccines. 2012;11(6):663-76. doi: 10.1586/erv.12.36.

6. Stamm WE, Norrby SR. Urinary tract infections: disease panorama and challenges. J Infect Dis. 2001;183 Suppl 1:S1-4. doi: 10.1086/318850.

7. Bertolino A, Mastucci E, Porro V, Corfiati L, Palermo $\mathrm{M}$, Ecari U, Ceccarelli G. Etizolam in the treatment of generalized anxiety disorder: a controlled clinical trial. J Int Med Res. 1989;17(5):455-60. doi: 10.1177/030006058901700507.

8. Junuzovic D, Hasanbegovic M, Zvizdic S, Hamzic S, Zunic L. The connection between endourological 
Annals of International Medical and Dental Research

E-ISSN: 2395-2822 | P-ISSN: 2395-2814

Vol-8, Issue-1 | January-February 2022

DOI: 10.53339/aimdr.2022.8.1.5

Page no- 31-37 | Section- Research Article (Microbiology)

procedures and occurrence of urinary infections. Mater Sociomed. 2014;26(4):237-41. doi: 10.5455/msm.2014.237-241.

9. Gupta K, Hooton TM, Stamm WE. Increasing antimicrobial resistance and the management of uncomplicated community-acquired urinary tract infections. Ann Intern Med. 2001;135(1):41-50. doi: 10.7326/0003-4819-135-1-200107030-00012.

10. Byington CL, Rittichier KK, Bassett KE, Castillo H, Glasgow TS, Daly J, Pavia AT. Serious bacterial infections in febrile infants younger than 90 days of age: the importance of ampicillin-resistant pathogens. Pediatrics. 2003;111(5 Pt 1):964-8. doi: 10.1542/ peds.111.5.964.
11. Gupta V, Yadav A, Joshi RM. Antibiotic resistance pattern in uropathogens. Indian J Med Microbiol. 2002;20(2):96-8.

12. Goldstein FW. Antibiotic susceptibility of bacterial strains isolated from patients with communityacquired urinary tract infections in France. Multicentre Study Group. Eur J Clin Microbiol Infect Dis. 2000;19(2):112-7. doi: 10.1007/s100960050440.

Source of Support: Nil, Conflict of Interest: None declared 